\title{
Characterization of an orthotopic gastric cancer mouse model with lymph node and organ metastases using bioluminescence imaging
}

\author{
HAI-YI FENG ${ }^{1-4 *}$, YUNPENG ZHANG $^{1 *}$, HAI-JUN LIU ${ }^{2,3}$, XIAO DONG $^{2,3}$, SI-CONG YANG $^{2,3}$,

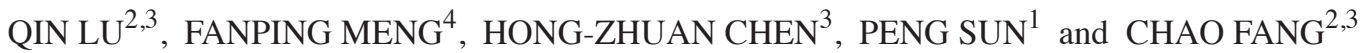 \\ ${ }^{1}$ Department of General Surgery and ${ }^{2}$ Hongqiao International Institute of Medicine, Shanghai Tongren Hospital, \\ Shanghai Jiao Tong University School of Medicine, Shanghai 200336; ${ }^{3}$ Department of Pharmacology \\ and Chemical Biology, Institute of Medical Sciences, Shanghai Jiao Tong University School of Medicine, \\ Shanghai 200025; ${ }^{4}$ College of Medicine, Yanbian University, Yanji, Jilin 133002, P.R. China
}

Received December 14, 2017; Accepted July 16, 2018

DOI: $10.3892 / \mathrm{ol} .2018 .9313$

\begin{abstract}
Lymph node (LN) metastasis of gastric cancer (GC) is the strongest prognostic indicator for this disease; however, the majority of the $\mathrm{LN}$ metastasis profiles of GC remain unknown, which notably hinders the therapeutic efficacy in clinic. In the present study, an orthotopic model of human GC was established for investigation of time-dependent LN metastasis patterns in mice. Luciferase-expressing NCI-N87 human GC cells were injected into the subserosa of the gastric body, resulting in a tumor formation rate of $100 \%$. LN metastasis at four different anatomical positions in the abdomen were characterized until week 10 after tumor cell injection using sensitive bioluminescence imaging and histopathological analyses. Skip LN metastases were observed at later stages (weeks 8-10) of the experiment. Metastases in other major organs, including liver, spleen and lung, were also examined. Characterization of this orthotopic GC model and metastasis patterns in LNs and major organs should aid in the preclinical GC research regarding the metastatic mechanism and drug development.
\end{abstract}

Correspondence to: Dr Peng Sun, Department of General Surgery, Shanghai Tongren Hospital, Shanghai Jiao Tong University School of Medicine, 1111 Xianxia Road, Shanghai 200336, P.R. China E-mail: sp2082@shtrhospital.com

Dr Chao Fang, Hongqiao International Institute of Medicine, Shanghai Tongren Hospital, Shanghai Jiao Tong University School of Medicine, 1111 Xianxia Road, Shanghai 200336, P.R. China

E-mail: fangchao32@sjtu.edu.cn

*Contributed equally

Key words: gastric cancer, orthotopic model, lymph node, metastasis, bioluminescence imaging

\section{Introduction}

Gastric cancer (GC) is the fourth most common cancer type and the second leading cause of cancer-induced mortality worldwide (1). Lymph node (LN) metastasis is the primary spread route and considered the strongest prognostic indicator of GC (2). Even for patients with early stage GC, the frequency of LN metastasis is estimated to be up to $20 \%$ (1).

Adequate surgical resection is considered the most favorable therapeutic option for GC (3-5). For this procedure, complete resection of the primary cancer with tumor-free surgical margins of $\geq 4 \mathrm{~cm}$ and adequate lymphadenectomy are required. D2 lymphadenectomy [perigastric (D1) plus coeliac artery and its branches] is generally recommended, based on associated clinical trials demonstrating the survival advantages of this surgery type $(1,6)$; however, the postsurgical morbidity and mortality outcomes are not always acceptable if the procedures are not performed by experienced surgeons. Additionally, surgeons in Western countries, including USA, UK and the Netherlands prefer using conservative D0 or D1 resection or modified D2 resection (without routine pancreatectomy and splenectomy) due to the negative results of two earlier randomized trials comparing D1 and D2 resection from the UK and the Netherlands $(1,6,7)$. These differences in lymphadenectomy are primarily attributable to respective history-based clinical experience and comprehensive evaluation of the benefits and risks of the procedure.

Precise resection of the LN with metastasis, while sparing healthy nodes, should present the most reliable criteria. This approach not only allows the removal of tumor cells in vivo as thoroughly as possible, in order to prevent relapse, but also avoids excessive lymphadenectomy, which notably affects the benefits to patients; however, the majority of $\mathrm{LN}$ metastasis patterns of GC remain unknown. Previous studies indicated that $\mathrm{LN}$ metastasis patterns in GC are notably complex, due to aberrant lymphatic drainage patterns with frequent occurrence of skip LN metastasis $(8,9)$. Micrometastases or isolated tumor cells in the LN increase the challenge of treating GC (2). 
Limited preclinical studies have focused on LN metastasis in GC to date (10-12). Furthermore, previous studies only reported the frequency of $\mathrm{LN}$ metastasis and did not highlight the anatomic positions of metastatic LNs. Clarification of the mechanisms underlying LN metastasis of GC may also be of significant value in directing clinical practice.

In the present study, an orthotopic human GC model was established in nude mice with luciferase-expressing NCI-N87 human GC cells. Cells were carefully injected into the subserosa of the gastric body, following which tumors formed and grew. Tumor development and metastasis in the LN and other major organs were monitored with the aid of sensitive bioluminescence imaging. The patterns of LN metastasis at four anatomic positions in the abdominal cavity were further characterized.

\section{Materials and methods}

Cell line and mouse experiments. The luciferase-expressing human GC cell line NCI-N87-Luc was obtained from Shanghai Biomodel Organisms Center Co., Ltd. (Shanghai, China). Cells were cultured in Dulbecco's modified Eagle's medium (DMEM) supplemented with $10 \%$ fetal bovine serum and antibiotics $(100 \mathrm{mg} / \mathrm{ml}$ streptomycin and $100 \mathrm{U} / \mathrm{ml}$ of penicillin) (all from (Thermo Fisher Scientific, Inc., Waltham, MA, USA) at $37^{\circ} \mathrm{C}$ in a humidified incubator with $5 \% \mathrm{CO}_{2}$.

A total of 100 female BALB/c nude mice (4-5 weeks old, $\sim 20 \mathrm{~g}$ ) were provided by the Shanghai Laboratory Animal Center (Chinese Academy of Sciences, Shanghai, China). The mice were fed in a specific pathogen-free environment at room temperature, with free access to food and water and 12:12 h light/dark cycle. The animal experiment designed in the present study was approved by the ethical committee of Shanghai Jiao Tong University School of Medicine (Shanghai, China).

Orthotopic injection of NCI-N87-Luc cells. The orthotopic injection technique was performed using a previously reported method (13). The mouse abdomen was opened via a midline incision under aseptic conditions, and the stomach was exteriorized. A volume of $40 \mu \mathrm{l}$ DMEM containing $5 \times 10^{6}$ NCI-N87-Luc cells was injected into the subserous layer of the middle of the stomach using a $100 \mu \mathrm{l}$ syringe and $30 \mathrm{G}$ needle. A cotton swab was pressed against the injection site for $\geq 20 \mathrm{sec}$ to prevent tumor cell leakage into the peritoneal cavity. Subsequently, the stomach was returned to the peritoneal cavity, and peritoneum and skin sutured sequentially. The feasibility of the injection method was confirmed using trypan blue as an indicator, which demonstrated an accurate location of the injected dye in the subserosa of the gastric wall.

Bioluminescence imaging of orthotopic tumor growth and metastasis. Female BALB/c nude mice (4-5 weeks old) were used for animal studies. At 4, 6, 8 and 10 weeks after tumor cell injection, mice $(n=5)$ were intraperitoneally injected with $200 \mu \mathrm{l}$ D-luciferin solution $(150 \mathrm{mg} / \mathrm{kg}$ body weight, J\&K Scientific Ltd, Shanghai, China). Subsequently, mice were anesthetized with $2 \%$ isoflurane, and at $8 \mathrm{~min}$ after D-luciferin injection, placed in the Xenogen IVIS 200 (PerkinElmer, Inc., Waltham, MA, USA) chamber with right lateral recumbency for bioluminescence imaging of the orthotopic NCI-N87-Luc tumor according to the manufacture's protocols. Body weights of mice were recorded weekly throughout the test. To detect metastasis in $\mathrm{LN}$ and other major organs at different time points, mice $(n=5)$ were sacrificed by cervical vertebra dislocation between week 3 and 10. LNs at four anatomical positions in the abdomen (14), including gastric LN (GLN), pancreaticoduodenal LN (PLN), mesenteric LN (MLN) and lumbar LN (LuLN), and other major organs, including the heart, liver, spleen, lung and kidney, were excised for ex vivo bioluminescence imaging. A metastasis heat map was generated using GraphPad Prism 7 software (GraphPad Software, Inc., La Jolla, CA, USA).

Histopathological assay. Excised LN and organs were fixed in $4 \%$ paraformaldehyde at room temperature overnight, embedded in paraffin and cut into $4 \mu \mathrm{m}$ sections. Subsequently the sections were stained with hematoxylin for $3 \mathrm{~min}$ and eosin for $2 \mathrm{~min}$ at room temperature. The sections were observed and photographed under upright metallurgical microscope with digital image capturing system (DP50; magnification, x40 or x200; Olympus Cooperation, Tokyo, Japan).

Statistical analysis. Data analysis was conducted with the GraphPad Prism 7.0 software (GraphPad Software, Inc., La Jolla, CA, USA). The quantified time-dependent bioluminescence signal of the tumors and also the mouse body weight are presented as mean \pm standard deviation.

\section{Results}

Establishment and characterization of an orthotopic model of $G C$. Trypan blue dye was used to confirm the reliability of the injection technique (Fig. 1A). Following the NCI-N87-Luc cell injection, a small white plaque was observed under the serosa of the gastric body (Fig. 1B). The cells formed an orthotopic tumor, which was detected via sensitive bioluminescence imaging (Fig. 1C). Tumors on the gastric wall were observed following opening the abdomen (Fig. 1D) or directly from the resected stomach (Fig. 1E). Ex vivo bioluminescence imaging highlighted the tumor position in the stomach (Fig. 1F). Histopathological assays further confirmed tumor formation and anatomical location of subserosa (Fig. 1G). All mice receiving this treatment grew tumors on the stomach, establishing a $100 \%$ success rate of the model.

Bioluminescence imaging of the growth of orthotopic gastric tumors. The growth of stomach tumors was monitored via bioluminescence imaging (Fig. 2A). Quantification of bioluminescence intensity demonstrated the time-dependent tumor growth until week 10 after tumor cell injections (Fig. 2B). During the same time-period, the body weight of the mice increased in the first 7 weeks. However, subsequent to week 7 the body weight of the mice declined (Fig. 2C), suggesting the involvement of harmful gastric tumors in the deterioration of the mice's health.

LN metastasis in the abdomen. Firstly, the four anatomical positions of LNs (GLN, PLN, MLN, and LuLN) were identified in the abdomen (Fig. 3A-C). H\&E staining further confirmed 
A

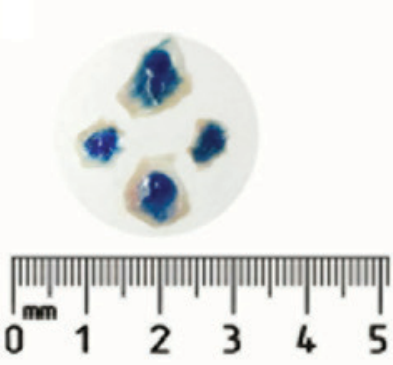

B

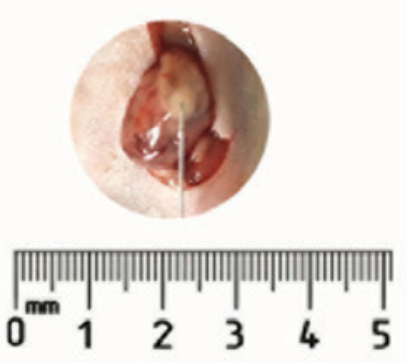

C

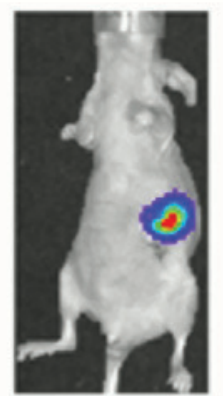

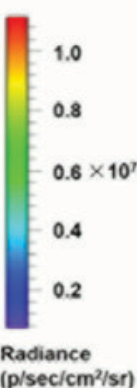
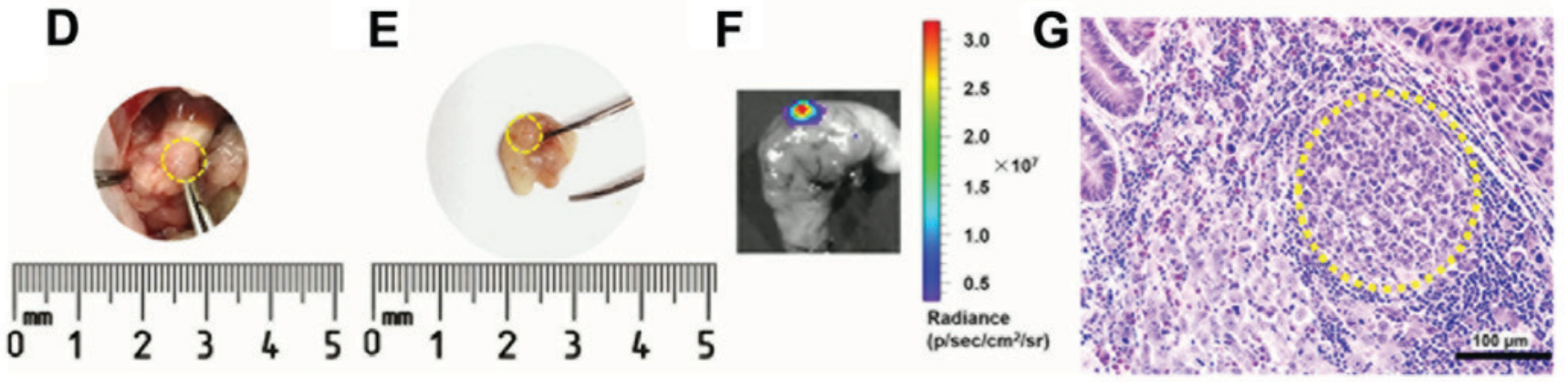

Figure 1. Establishment of an orthotopic model of gastric cancer. (A) Trypan blue was used as an indicator to demonstrate that the injection of NCI-N87-Luc cells into the gastric wall was feasible. It can be observed that the dye was located under the serosa. (B) Orthotopic tumor cell injections. A small white plaque was observed under the serosa of the gastric body. (C) At day 14 after tumor cell injection, bioluminescence imaging demonstrated the formation of the orthotopic tumor. (D) The ex vivo view of the stomach with the tumor following the abdomen being opened. (E) The excised stomach with the tumor. (F) Ex vivo bioluminescence imaging at day 20 after tumor cell injection. $(\mathrm{G})$ Hematoxylin and eosin staining of the stomach with the gastric tumor. The malignant lesion of the tumor was circled with the yellow dashed line.
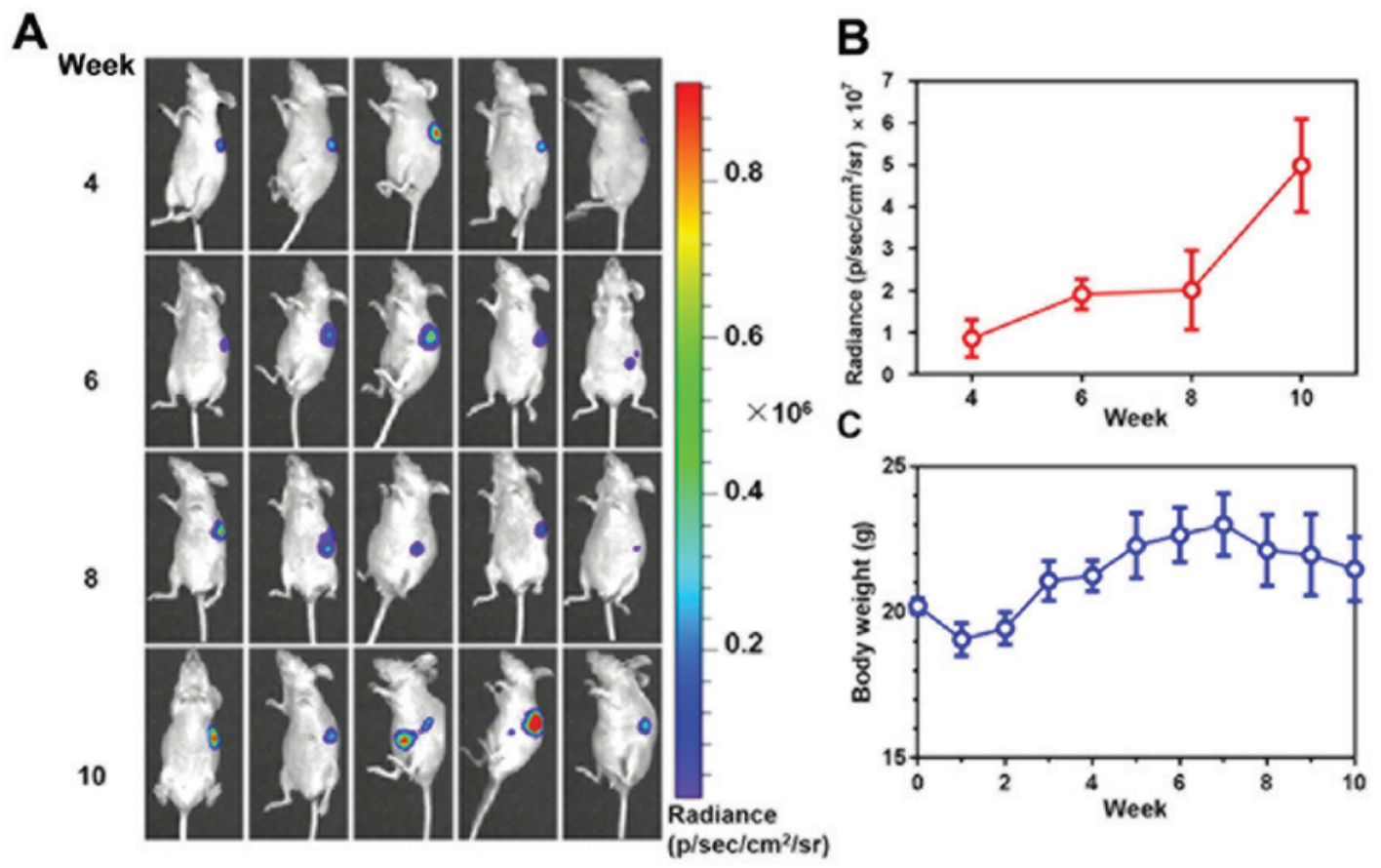

Figure 2. In vivo bioluminescence imaging of the orthotopic gastric tumor growth. (A) The NCI-N87-Luc cells were injected into the subserosa of the gastric wall. At week 4, 6, 8 and 10 after injection, the mice $(n=5)$ were imaged to observe the growth of the tumors. (B) The quantified bioluminescence signal demonstrated the time-dependent growth of the tumor. (C) Body weight observation.

the presence of LNs, and not adipose tissues or omentum, at these positions (Fig. 3D).

From week 3, LNs of mice $(n=5)$ were resected for ex vivo bioluminescence imaging to investigate the metastasis patterns (Fig. 4A). The overall LN metastasis patterns from weeks 3-10 were summarized in a heat map (Fig. 4B). Histopathological examination with bioluminescence imaging further confirmed the presence of metastatic tumor cells in the LNs (Fig. 4C). This demonstrated that no metastasis occurred in the LN at week 3 and 4. At week 5, GLN of one mouse was 


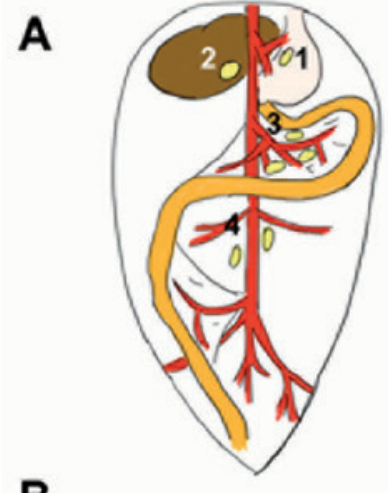

B
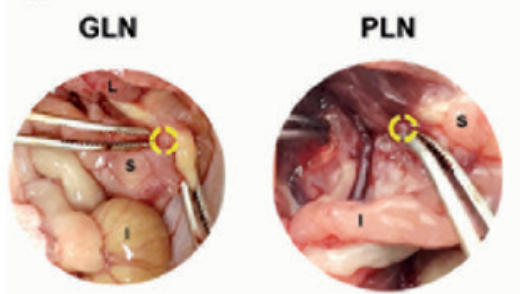

\begin{tabular}{|c|c|}
\hline Positions & Description \\
\hline $\begin{array}{l}\text { 1. Gastric LN } \\
\text { (GLN) }\end{array}$ & $\begin{array}{l}\text { At the lesser curvature of the stomach, } \\
\text { single or a pair of tiny } \mathrm{LN}\end{array}$ \\
\hline $\begin{array}{l}\text { 2. Pancreaticoduodenal LN } \\
\text { (PLN) }\end{array}$ & $\begin{array}{l}\text { At the superior border of pancreatic, } \\
\text { single or a pair of spherical LN, close to } \\
\text { the portal vein }\end{array}$ \\
\hline $\begin{array}{l}\text { 3. Mesenteric LN } \\
\text { (MLN) }\end{array}$ & $\begin{array}{l}\text { In the mesocolon, the largest LNs in the } \\
\text { nude mice, stretching like an earthworm } \\
\text { to mesenteric adipose tissue }\end{array}$ \\
\hline $\begin{array}{l}\text { 4. Lumbar LN } \\
\text { (LuLN) }\end{array}$ & $\begin{array}{l}\text { A pair or more on both sides of } \\
\text { abdominal aorta }\end{array}$ \\
\hline
\end{tabular}

C
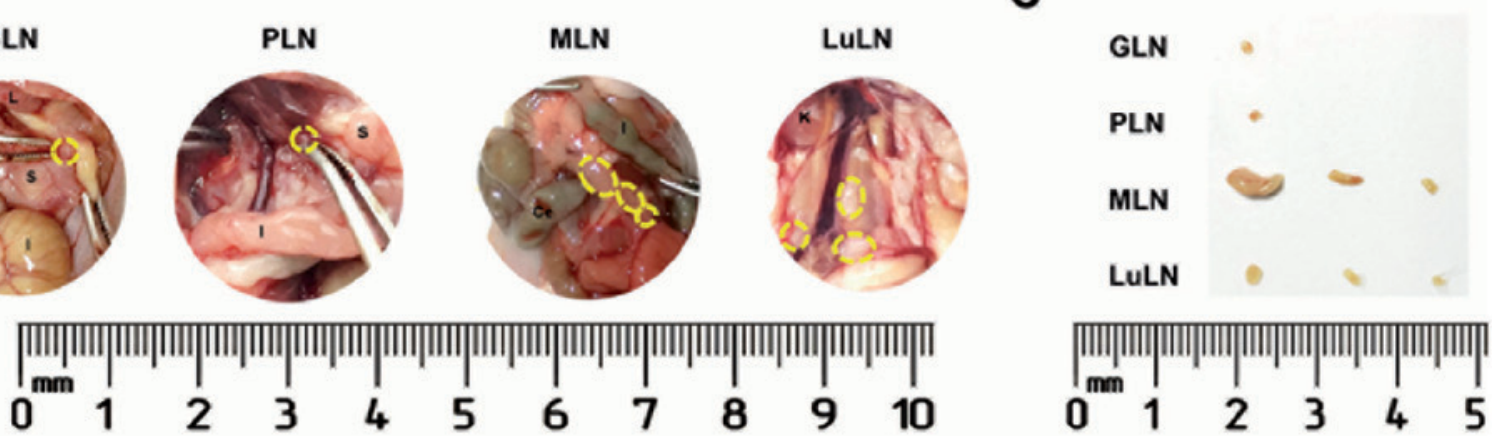

D
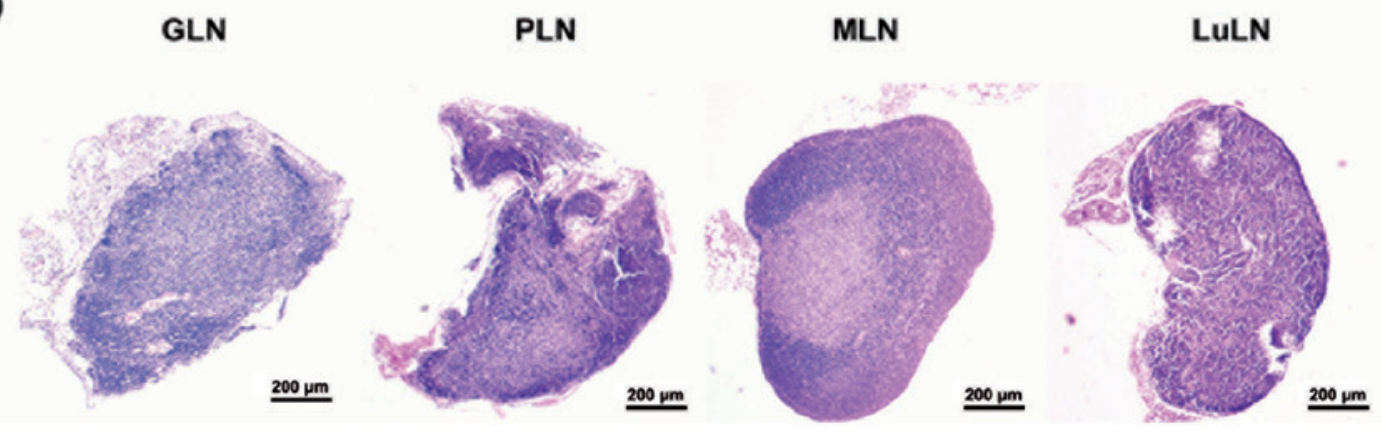

Figure 3. Identification of LNs at different anatomical position in the abdomen. (A) Schematic anatomical positions of the LNs (GLN, PLN, MLN and LuLN) detected in the present study. At 10 weeks after tumor cell injection, the LNs at various positions were indicated following (B) exploratory laparotomy, and (C) then were excised and arranged on clean paper. The LNs were indicated with yellow circles. (D) Hematoxylin and eosin staining of the resected LNs at different positions. L, liver; S, stomach; I, intestine; Ce, cecum; K, kidney; LN, lymph nodes; GLN, gastric LN; PLN, pancraticoduodenal LN; MLN, mesenteric LN; LuLN, lumbar LN.

positive for metastasis, based on the bioluminescence signal. At week 6, metastasis was observed at PLN, MLN and LuLN in one mouse, indicating distant spreading. From weeks 7-9, metastasis occurred in a greater number of mice and spread into LNs at various anatomical positions. At week 10, all tested mice exhibited positive bioluminescence signals in LNs. It is also notable that the metastasis frequency for GLN, MLN and LuLN decreased from $40 \%$ at week 7 to $20 \%$ at week 8 . This phenomenon may be ascribed to the individual differences of the mice at each week, which may be avoided if a greater number of animals $(n>5)$ were examined at each time point. Additionally, it can be concluded, though this observation, that the metastasis frequency for GLN, MLN and LuLN varied in the range of $20-40 \%$ in this duration.

Major organ metastasis. Organ metastasis was observed at week 5 (the same time as LN metastasis). One mouse exhibited metastasis to the liver and one to the spleen (Fig. 5); however, the bioluminescence signals detected were weak, indicating the metastasis of only a small number of tumor cells. From weeks 6-10, the spleen and liver remained the primary metastatic organs. Lung metastasis was only observed in one mouse at week 7. It also demonstrated a liver metastasis frequency of $20 \%$ at week 5 and 6 , which reduced to $0 \%$ at week 7 and 8 . It is notable that the observations at each week were from different mice. If a greater number of animals $(n>5)$ were included for each week, the influence of animal individual differences on the metastasis frequency may be avoided. These observations also indicated that the liver metastasis were not frequent in the first 8 weeks in the present model.

\section{Discussion}

Treatment of GC primarily depends on the management of LN metastasis; however, unique metastasis patterns, such as skip LN metastasis, cause a notable challenge to effective treatments. 

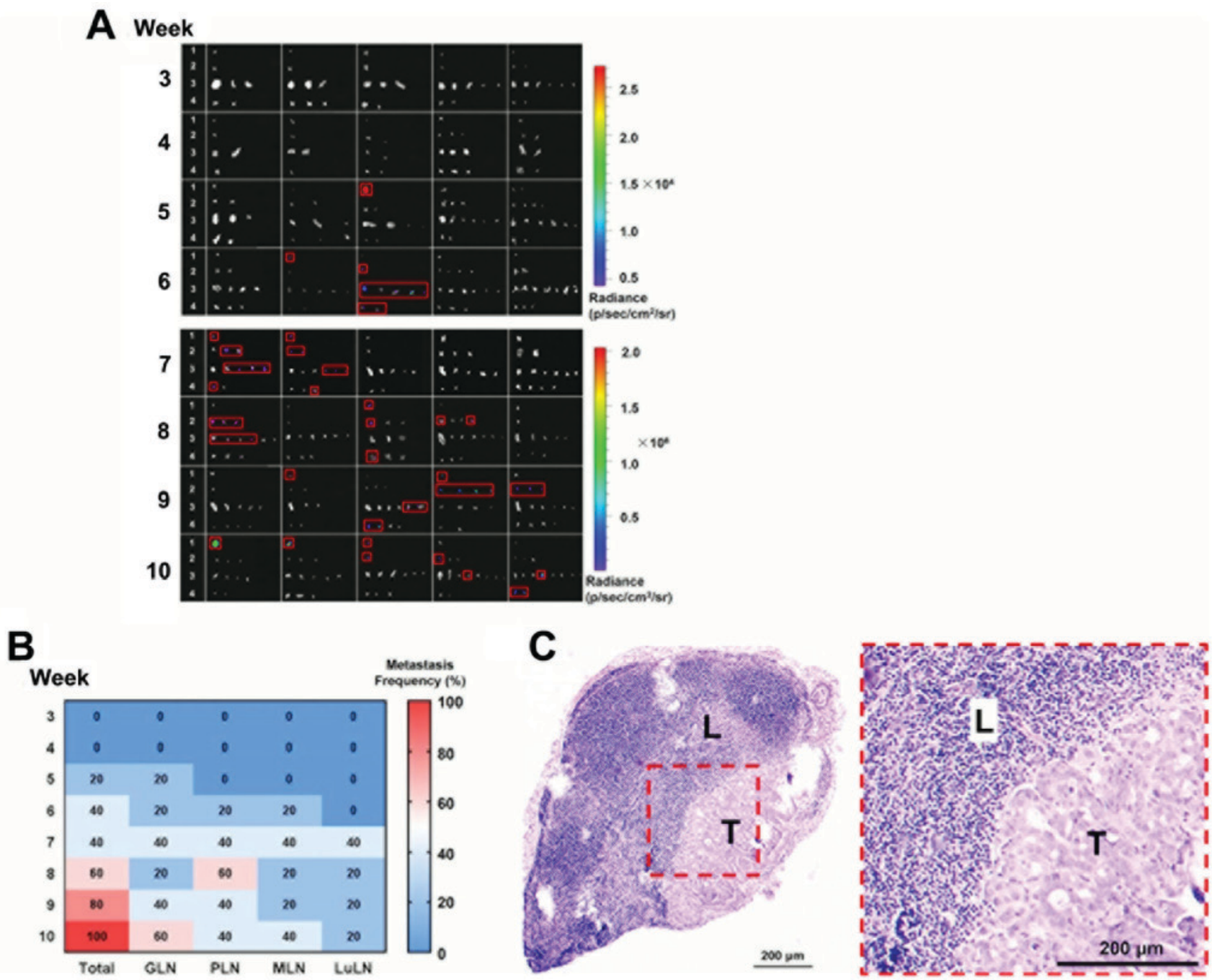

Figure 4. LN metastasis of orthotopic gastric cancer model. From weeks 3-10, mice ( $\mathrm{n}=5$ for each week) were sacrificed, and the LNs (1, GLN; 2, PLN; 3, MLN; and 4, LuLN) were resected for metastasis detection. Note that the images presented for each week are not from the same mouse. (A) LNs metastasis detected using the bioluminescence imaging. Each black rectangle indicates the LNs of one mouse. The red rectangles indicated the identified metastatic LNs. (B) The time-dependent metastasis frequencies of LNs at different sites were summarized in the heat map. (C) Hematoxylin and eosin staining of the resected metastatic GLN after 6 weeks. L, lymphocyte; T, tumor cells; LN, lymph nodes; GLN, gastric LN; PLN, pancraticoduodenal LN; MLN, mesenteric LN; LuLN, lumbar LN.

Micrometastasis and isolated tumor cells in regional LNs also pose a serious threat to prognosis. The complex profile of LN metastasis of GC remains largely unknown, which is partially responsible for the controversy regarding the range of lymphadenectomy. Previous preclinical studies have been limited to the time-dependent frequency of LN metastasis in GC $(12,13,15)$. However, to the best of our knowledge, no studies have been conducted regarding LN metastasis patterns at specific anatomical positions, which may be beneficial for a surgeon to perform precise surgery.

In the present study, an orthotopic GC model was established via injection of NCI-N87-Luc cells into the subserosa of the gastric body of nude mice. The present method appeared to be reliable and reproducible, resulting in a tumor formation rate of $100 \%$. Additionally, the present procedure could overcome the risk of tumor cells shedding into the peritoneal cavity, which occurs with other methods, including fixing the donor tumor fragment into the recipient gastric wall using tissue adhesive (15). The metastasis was identified using the sensitive bioluminescence imaging, which can detect 100 tumor cells in vitro (data not shown) and avoid the false negative results when performing traditional serial sectioning and histopathological examinations (2). It demonstrated that there was no detectable metastasis at week 3 and 4 in LNs and the major organs (Figs. 4 and 5), indicating that the metastasis was probably from the primary GC, which requires a long time for the tumor cells to colonize and form detectable foci.

The time-dependent metastasis patterns in the four LN positions (GLN, PLN, MLN and LuLN) in the abdomen were characterized. As expected, the frequency of metastasis increased with time following tumor cell injection. Notably, skip metastasis was observed in mice at the later stages (weeks 8-10) of the experiment period, demonstrating a discontinuous spread of malignancy in the $\mathrm{LN}$, providing further evidence of the complexity of LN metastasis in GC.

The frequency and extent of metastasis in major organs other than LN was significantly reduced, which may be partially attributed to the well-differentiated property of NCI-N87 cells. Further studies are warranted to investigate the metastatic behavior of orthotopic poorly-differentiated gastric 

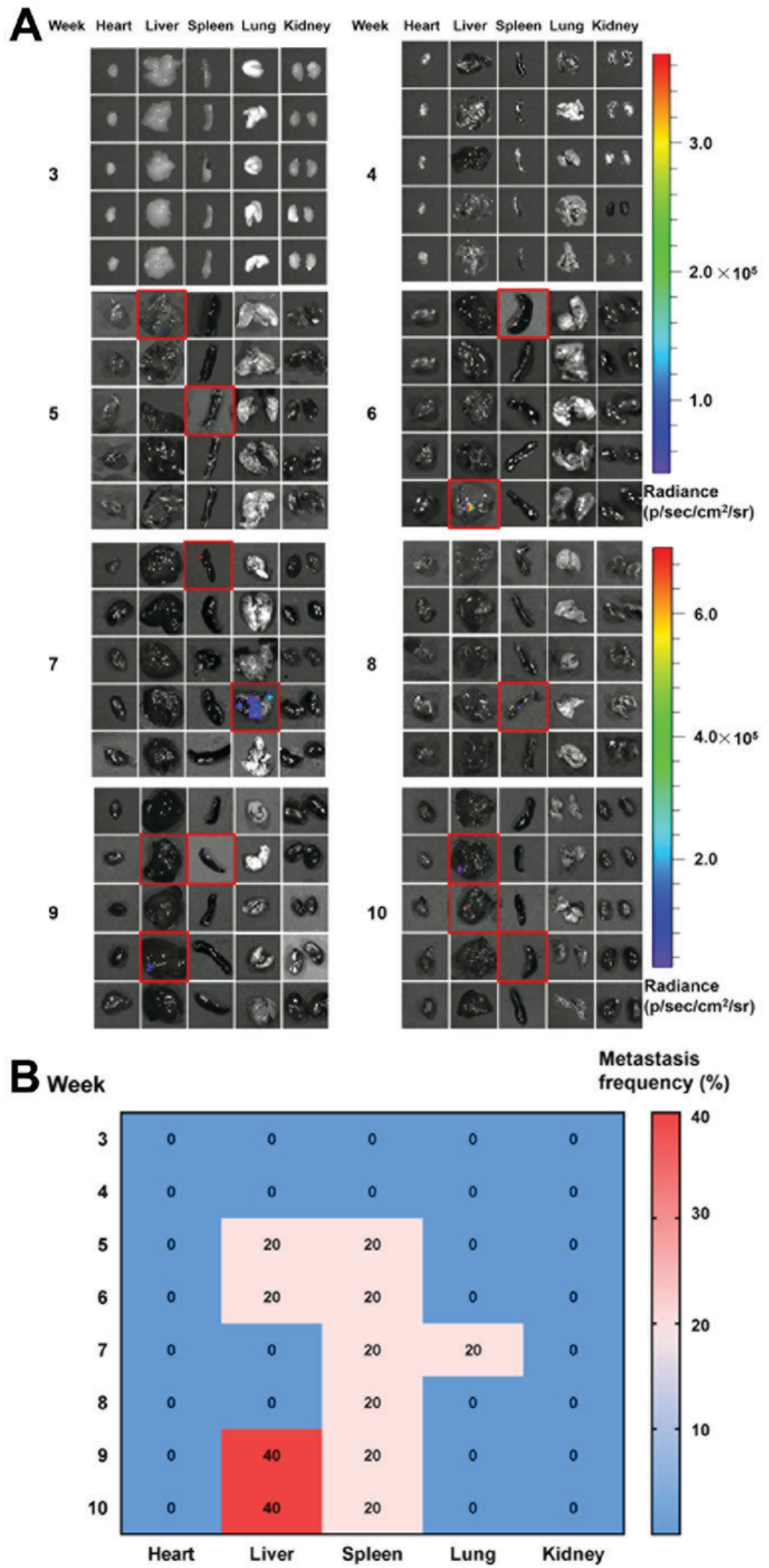

Figure 5. The metastasis in major organs of the gastric cancer mice model. From week $3-10$, mice ( $n=5$ for each week) were sacrificed and the major organs, including the heart, liver, spleen, lung and kidney, were resected for metastasis detection. Note that the images presented for each week are not from the same mouse. (A) The metastases were detected using the bioluminescence imaging. The red rectangles indicated the identified metastatic organs. Each row represents one mouse. (B) The time-dependent metastasis frequencies in the major organs were summarized in the heat map. 
tumor types, including the AGS $(16,17)$ and MKN-45 $(18,19)$ cell lines, or tumor types with diverse molecular classifications, including HER2-positive cancer types $(1,20)$.

In conclusion, GC metastasis in LNs and major organs was characterized with the aid of an established orthotopic mouse model. Time-dependent metastasis patterns at different anatomical positions in the abdomen are presented. The present model with characterized LN and organ metastasis may be effectively used for preclinical GC research regarding the metastatic mechanism and drug development.

\section{Acknowledgements}

We thank Dr Jing Zhou (Department of Experimental Animal Science, Shanghai Jiao Tong University School of Medicine) for assisting with IVIS imaging testing. We also thank Dr Lin Zheng (Department of Pathology, Shanghai Jiao Tong University School of Medicine) for assisting with the histopathological assay.

\section{Funding}

The present study is supported by National Natural Science Foundation of China (grant nos. 81401958, 81572998 and 81773274), Shanghai Municipal Commission of Health and Family Planning (grant no. ZK2015A25), Shanghai Changning Commission of Health and Family Planning (grant no. YXMZK004) and Shanghai Municipal Science and Technology Commission (grant nos. 14ZR1431700 and 16520710700), 'Shu Guang' Program of Shanghai Education Development Foundation and Shanghai Municipal Education Commission (grant no. 16SG13), Shanghai Collaborative Innovation Center for Translational Medicine (grant no. TM201731).

\section{Availability of data and materials}

The datasets used and analyzed during the present study are available from the corresponding author on reasonable request.

\section{Authors' contributions}

HF, PS and CF conceived and designed the experiments. HF, YZ, HL, XD, SY and QL performed the experiments. HF and CF collected and analyzed the data. FM and HZC provided suggestions and technical support on the project. $\mathrm{HF}$ and $\mathrm{CF}$ wrote the manuscript. PS and CF supervised the project. All authors read and approved the final manuscript.

\section{Ethics approval and consent to participate}

The animal experiments designed in this study were approved by the ethical committee of Shanghai Jiao Tong University School of Medicine.

\section{Patient consent for publication}

Not applicable.

\section{Competing interests}

The authors declare that they have no competing interests.

\section{References}

1. Van Cutsem E, Sagaert X, Topal B, Haustermans K and Prenen H: Gastric cancer. Lancet 388: 2654-2664, 2016.

2. Jeuck TL and Wittekind C: Gastric carcinoma: Stage migration by immunohistochemically detected lymph node micrometastases. Gastric Cancer 18: 100-108, 2015.

3. Songun I, Putter H, Kranenbarg EM, Sasako M and van de Velde CJ: Surgical treatment of gastric cancer: 15 -year follow-up results of the randomised nationwide Dutch D1D2 trial. Lancet Oncol 11: 439-449, 2010.

4. Ajani JA, D'Amico TA, Almhanna K, Bentrem DJ, Chao J, Das P, Denlinger CS, Fanta P, Farjah F, Fuchs CS, et al: Gastric cancer, version 3.2016, NCCN clinical practice guidelines in oncology. J Natl Compr Canc Netw 14: 1286-1312, 2016.

5. Smyth EC, Verheij M, Allum W, Cunningham D, Cervantes A, Arnold D and ESMO; Guidelines Committee: Gastric cancer: ESMO Clinical Practice Guidelines for diagnosis, treatment and follow-up. Ann Oncol 27 (Suppl 5): v38-v49, 2016.

6. Shen L, Shan YS, Hu HM, Price TJ, Sirohi B, Yeh KH, Yang YH, Sano T, Yang HK, Zhang X, et al: Management of gastric cancer in Asia: Resource-stratified guidelines. Lancet Oncol 14: e535-e547, 2013.

7. Ajani JA, Lee J, Sano T, Janjigian YY, Fan D and Song S: Gastric adenocarcinoma. Nat Rev Dis Primers 3: 17036, 2017.

8. Kim DH, Choi MG, Noh JH, Sohn TS, Bae JM and Kim S: Clinical significance of skip lymph node metastasis in gastric cancer patients. Eur J Surg Oncol 41: 339-345, 2015.

9. Choi YY, An JY, Guner A, Kang DR, Cho I, Kwon IG, Shin HB, Hyung WJ and Noh SH: Skip lymph node metastasis in gastric cancer: Is it skipping or skipped? Gastric Cancer 19: 206-215, 2016.

10. Qiao R, Liu C, Liu M, Hu H, Liu C, Hou Y, Wu K, Lin Y, Liang J and Gao M: Ultrasensitive in vivo detection of primary gastric tumor and lymphatic metastasis using upconversion nanoparticles. ACS Nano 9: 2120-2129, 2015.

11. Furukawa T, Fu X, Kubota T, Watanabe M, Kitajima M and Hoffman RM: Nude mouse metastatic models of human stomach cancer constructed using orthotopic implantation of histologically intact tissue. Cancer Res 53: 1204-1208, 1993.

12. Fujihara T, Sawada T, Hirakawa K, Chung YS, Yashiro M, Inoue T and Sowa M: Establishment of lymph node metastatic model for human gastric cancer in nude mice and analysis of factors associated with metastasis. Clin Exp Metastasis 16: 389-398, 1998.

13. Hackl C, Man S, Francia G, Milsom C, Xu P and Kerbel RS: Metronomic oral topotecan prolongs survival and reduces liver metastasis in improved preclinical orthotopic and adjuvant therapy colon cancer models. Gut 62: 259-271, 2013.

14. Van den Broeck W, Derore A and Simoens P: Anatomy and nomenclature of murine lymph nodes: Descriptive study and nomenclatory standardization in BALB/cAnNCrl mice. J Immunol Methods 312: 12-19, 2006.

15. Bhargava S, Hotz B, Buhr HJ and Hotz HG: An orthotopic nude mouse model for preclinical research of gastric cardia cancer. Int J Colorectal Dis 24: 31-39, 2009.

16. Yin J, Cui Y, Li L, Ji J and Jiang WG: Overexpression of EPHB4 is associated with poor survival of patients with gastric cancer. Anticancer Res 37: 4489-4497, 2017.

17. Belkhiri A, Dar AA, Zaika A, Kelley M and El-Rifai W: t-Darpp promotes cancer cell survival by up-regulation of $\mathrm{Bcl} 2$ through Akt-dependent mechanism. Cancer Res 68: 395-403, 2008.

18. Guo B, Zhao Z, Wang Z, Li Q, Wang X, Wang W, Song T and Huang C: MicroRNA-302b-3p suppresses cell proliferation through AKT pathway by targeting IGF-1R in human gastric cancer. Cellular Physiol Biochem 42: 1701-1711, 2017.

19. Burgermeister E, Xing X, Röcken C, Juhasz M, Chen J, Hiber M, Mair K, Shatz M, Liscovitch M, Schmid RM and Ebert MP: Differential expression and function of caveolin-1 in human gastric cancer progression. Cancer Res 67: 8519-8526, 2007.

20. Boku N: HER2-positive gastric cancer. Gastric Cancer 17: 1-12, 2014. 